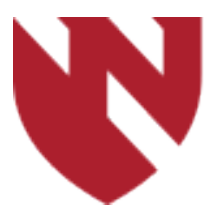

December 2019

\title{
Diagnostic Challenges in the Radiographic Distinction between Demyelinating Lesions and Primary Brain Neoplasms
}

\author{
Erin Cameron Smith \\ University of Nebraska Medical Center \\ Geetanjali Rathore \\ University of Nebraska Medical Center
}

Tell us how you used this information in this short survey.

Follow this and additional works at: https://digitalcommons.unmc.edu/gmerj

Part of the Higher Education Commons, and the Medicine and Health Sciences Commons

\section{Recommended Citation}

Cameron Smith, E., , Rathore, G. Diagnostic Challenges in the Radiographic Distinction between Demyelinating Lesions and Primary Brain Neoplasms. Graduate Medical Education Research Journal. 2019 Dec 13; 1(1). https://digitalcommons.unmc.edu/gmerj/vol1/iss1/53 
Diagnostic Challenges in the Radiographic Distinction between Demyelinating Lesions and Primary Brain Neoplasms

\section{Creative Commons License}

\section{c) (1)@ $\Theta$}

This work is licensed under a Creative Commons Attribution-Noncommercial-No Derivative Works 4.0 License. 
Methods: Data were collected using EPIC® starting 12 months prior to VNS replacement to Aspire ${ }^{\circledR}$ device to 12 months after implantation date in 3-month blocks. Seizure frequency before and after the VNS implantation as well as medication changes were identified from review of clinic notes. Cost was estimated based on the number of visits for ER, clinic, hospital, and ICU using hospital average cost data. As cost distributions are skewed to the right, we also calculated log total costs for the trend analysis using segmented model. For data analysis segmented Poisson regression was used to establish a trend in usage per quarter.
Results: The trend in Clinic usage was statistically flat $(\mathrm{p}>0.05)$ in the preplacement period while the trend in the post placement was downward with an estimate that for every quarter the natural log of the average number of clinic visits decreases by 0.29 . By one-year post placement, the average number of clinic visits had achieved the same level as the pre-placement period. The trends in ER and hospital usage were flat in both the pre and post placement periods. The median number of CPS in the year after placement of the Aspire 106 VNS was statistically smaller by $1(\mathrm{p}=0.0161)$ CPS than the median number of CPS in the year prior to placement.
Conclusion: Analysis of the above data yielded two main findings: there were no demonstrable cost savings within a 12-month period following VNS implantation, but the median burden of Complex Partial Seizures (CPS) within our cohort revealed a 50\% decline, which was statistically significant. However, overall costs post-implantation showed a consistent downward trajectory, with approximation to pre-implantation costs within the 12-month timeframe.

https://doi.org/10.32873/unmc.dc.gmerj.1.1.050

thalamus-midbrain junction just posterior to the red nucleus.

Conclusion: This case illustrates how a punctate single unilateral lesion can present with vertical supranuclear gaze palsy and horizontal eye movement abnormalities. https://doi.org/10.32873/unmc.dc.gmerj.1.1.051
Method: Characterize the eye movement deficits in this patient and correlate the MRI diffusion restriction findings.
Results: This patient presented with vertical gaze palsy and impaired adduction of the left eye. Impaired smooth pursuits up and down clinically localized to damage in the interstitial nucleus of Cajal. Impaired saccades up and down localized to the rostral interstitial nuclei of the medial longitudinal fasciculus. MRI showed diffusion restriction in the left

\section{Paroxysmal Autonomic Instability and Dystonia Following a Motor Vehicle Accident Matthew Purbaugh, Krishna Galla, Marco A. Gonzalez Castellon}

Mentor: Marco A. Gonzalez Castellon

Program: Neurology

Background: We were consulted a young adult patient who presented following a motor vehicle accident. The patient presented with diffuse axonal injury, hemorrhage of left basal ganglia increased intra-cranial pressure and other injuries. The patient was intubated, an intraventricular shunt had been removed and a 7-day course of Keppra prophylaxis had been finished. The patient was stable until the morning we were consulted.

Methods: On exam the patient had episodes of extensor posturing, diffuse dystonia and high frequency, low amplitude tremors, with autonomic instability limited to these spells.
The patient was found to have $3+$ reflexes throughout. Brain stem reflexes were intact. Stimuli caused extensor posturing, rigidity and autonomic instability. Continuous VEEG showed epileptiform activity that did not correlate with spells on video. Lab was non-specific expect for a reelevated CK. We loaded Keppra and prn diazepam. This failed to control the symptoms.

Results: We arrived at a diagnosis of paroxysmal autonomic instability and dystonia (PAID) syndrome. Treatment with clonazepam, Gabapentin, baclofen and propranolol stabilized the autonomic system and resolved the dystonia. The CK began to drop and the patient began to respond to commands.
Conclusions: PAID most commonly affects young persons following severe TBI. Potentially caused by disinhibition of sympathoexcitatory regions leading to cortically provoked catecholamine surges causing autonomic instability. Dystonia is secondary to disruption of the pontine and vestibular nuclei. PAID should on the differential diagnosis of TBI patients who are not responding to typical treatments. We had success treating with Clonazepam, gabapentin, baclofen and propranolol. https://doi.org/10.32873/unmc.dc.gmerj.1.1.052

\section{Diagnostic Challenges in The Radiographic Distinction Between Demyelinating Lesions and Primary Brain Neoplasms}

Erin L. Smith and Geetanjali Rathore

Mentor: Geetanjali Rathore

Program: Neurology

Introduction: The differentiation between demyelinating lesions and primary brain neoplasms is difficult based on radiography alone, resulting in misdiagnoses and delay in treatment. Here we present two cases with MRI abnormalities suggestive of either demyelination or neoplasm. Case 1 was treated with steroids for a demyelinating process, but was found on biopsy to have an anaplastic astrocytoma that was unresectable by the time of diagnosis. Case 2 was thought to be glioma but was later determined to be tumefactive demyelination. In both cases, there were delays due to the limitations of MR imaging.
Methods: The cases of the two patients were reviewed via EMR. A literature review investigated the most sensitive and specific non-invasive diagnostic modalities for diagnosing demyelinating lesions or primary brain neoplasms.

Results: Review of recent literature shows that conventional MR sequences have 
an established sensitivity of $80.9 \%$ and specificity of $57.1 \%$ in differentiating high-grade gliomas from tumefactive demyelinating lesions. Some studies suggest that even specimen biopsies may be inconclusive, as there are many histopathologic similarities between tumefactive demyelinating lesions and high-grade gliomas. Diffusion tensor imaging (DTI), though not yet utilized in common practice, can be highly sensitive in differentiating these lesions.
Conclusions: For both patient scenarios, MR imaging was insufficient to make a definitive diagnosis, resulting in a delay in treatment. The cases and literature review demonstrate the diagnostic challenges of these conditions using standard MRI alone, and raise awareness of DTI and other non-invasive modalities that may become integrated into future clinical practice.

https://doi.org/10.32873/unmc.dc.gmerj.1.1.053

\section{Utilization of Brain Imaging in Evaluating Patients with Psychogenic Nonepileptic Spells Danmeng Wei, Matthew Garlinghouse, Wenyang Li, Nicholas Swingle, Kaeli K. Samson, Olga Taraschenko}

Mentor: Olga Taraschenko

Program: Neurology

Background: Psychogenic non-epileptic spells (PNES) are paroxysmal movements or sensory events that resemble epileptic seizures but lack corresponding ictal electrographic changes. Patients are often referred for brain imaging tests which contribute to the high cost of care.

Methodology: This is a retrospective chart review of 225 adult patients diagnosed with PNES without epileptic seizures from 2012 to 2017. The frequency of the brain imaging tests prior to vEEG was assessed across all semiological classes and was correlated with other clinical characteristics.

Result: The most prevalent PNES events were characterized by semi-rhythmic small amplitude movements in the extremities (32\%) followed by those resembling clonictonic seizures $(27.7 \%)$. Patients with sensory changes had more imaging tests than those with primitive gesturing and axial posturing. Patients with 3 or more psychiatric disorders had more combined MRIs and CTs prior to diagnosis than patients with two or fewer psychiatric co-morbidities ( $\mathrm{p}=0.03)$.

Conclusion: The frequency of brain imaging obtained prior to the definitive diagnosis of PNES is influenced by semiology and the psychiatric health of patients. The PNES with minimal paroxysmal movements in the settings of multiple psychiatric co-morbidities represent particularly challenging patient phenotype which is linked to excessive referrals for brain imaging.

https://doi.org/10.32873/unmc.dc.gmerj.1.1.054

\section{Disseminated Strongyloides Causing Diffuse Alveolar Hemorrhage Mollie Brittan, Brian Boer, Deepak Chandra}

\section{Mentor: Brian Boer}

Program: Internal Medicine

Case presentation: An elderly patient with a past medical history of leukocytoclasic vasculitis on high dose steroids presented with altered mental status. The patient was found to be in septic shock and was admitted to an intensive care unit. The patient was started on broad spectrum antibiotics but continued to decline and developed hemoptysis along with hypoxic respiratory failure requiring intubation. Bronchoscopy revealed frank bronchial and alveolar hemorrhage. Bronchoscopy fluid was sent for microscopy and it revealed nematodes consistent with Strongyloides. The patient was started on

\section{Was It the Beer or the Burgers?} Ian Cormier, Craig Baumgart, Michael Smith

\section{Mentor: Michael Smith \\ Program: Internal Medicine}

Acute pancreatitis is a commonly encountered diagnosis and is the most common gastrointestinal reason for hospitalization. Approximately $75 \%$ of cases of acute pancreatitis are caused by gallstones oral Ivermectin and Albendazole but failed to improve on this regimen and was switched to subcutaneous Ivermectin after FDA approval. The patient ultimately improved and was discharged.

Discussion: Strongyloides stercolis is a nematode that can cause disease in humans. Strongyloides infection can involve cutaneous, pulmonary and gastrointestinal systems. This organism has a unique lifecycle where the parasite enters the host often through the skin and migrates to both the gastrointestinal and pulmonary systems at various points during its lifecycle. This unique life cycle also involves gastrointestinal autoinfection. Autoinfection occurs when a human is infected with a helminth (i.e.
Strongyloides) and the whole parasite's lifecycle takes place within the human host. This process is exaggerated in humans when cell mediated immunity is impaired with immunosuppression (in the case of our patient with corticosteroids). It has the ability to spread to multiple organs during the hyperinfection stage which can cause multisystem organ failure.

Conclusion: Diffuse alveolar hemorrhage caused by disseminated Strongylioides has a high mortality rate and treatment with subcutaneous Ivermectin contributed to her survival as the oral formulation had poor absorption due to gastrointestinal involvement of the parasite.

https://doi.org/10.32873/unmc.dc.gmerj.1.1.055 or alcohol consumption. In contrast, hypertriglyceridemia only accounts for $2-4 \%$ of acute pancreatitis cases.

A middle-aged patient with a history of recurrent acute pancreatitis secondary to alcohol abuse was admitted for acute epigastric abdominal pain consistent with his prior episodes of pancreatitis. Workup was significant for multiple electrolyte derangements and laboratory workers commented on the patient's serum appearing lactescent. Repeat point-of-care testing revealed normal electrolyte levels. A lipid panel revealed profound hypertriglyceridemia and the patient was treated with an insulin 